\title{
La conservación de los valores cromáticos de la arquitectura vernácula: casos de las rancherías Wayúu
}

\author{
Mariolly Davila Cordido y Caterina Macchi Jánica
}

\begin{abstract}
Resumen La incidencia de la luz sobre la tierra y la posición geográfica juegan un papel importante en la identidad del lugar. Tener en cuenta este hecho es esencial para intervenir y comprender lo tradicional de la arquitectura vernácula del Caribe colombiano. La arquitectura tradicional vernácula tiene una serie de características únicas, como sus propios métodos constructivos tradicionales que se yuxtaponen con su entorno geográfico. Esto genera auténticas y creativas paletas de color que se pueden ver en la cultura de los grupos indígenas de esa zona. Hoy en día, la arquitectura vernácula ocupa un lugar muy definido en relación a los criterios de procedimientos, metodologías de intervención y rehabilitación del Patrimonio Costero, ya que es una actividad espontánea de transmisión oral.
\end{abstract}

Palabras clave: Arquitectura vernácula, paletas de color, patrimonio colombiano, Ranchería Wayúu

\section{The conservation of the chromatic values of vernacular architecture: cases of the rancherias Wayúu}

Abstract: The incidence of light on the earth and geographical position play an important role, illustrating the identity of a place; it's essential to have in mind this situation to understand and act upon traditional vernacular architecture of the Colombian Caribbean. The traditional vernacular architecture has a series of unique characteristics, such as the very specific traditional construction methods that blend with its geographical environment. This generates authentic and creative color palettes that can be seen in the culture of the indigenous groups of that area. Nowadays, the vernacular architecture occupies an ambiguous place in relation to the criteria of procedures, intervention methodologies and rehabilitation of the Coastal Heritage, since it is a spontaneous activity of oral transmission

Keyword: Vernacular architecture, color palette, Colombian heritage, Rancheria Wayúu

\section{A conservação dos valores cromáticos da arquitetura vernacular: casos das rancherias Wayúu}

Resumo: A incidência da luz na terra e a posição geográfica desempenham um papel importante, ilustrando a identidade de um lugar; intervir e compor a arquitetura vernacular tradicional do Caribe colombiano. A arquitetura vernacular tradicional tem uma série de características únicas, como os métodos de construção tradicionais muito específicos que se misturam com o seu ambiente geográfico. Isso gera linguagens de cores autênticas e criativas que podem ser vistas na cultura dos grupos indígenas daquela área. Atualmente, a arquitetura vernacular ocupa um lugar ambíguo em relação aos critérios de procedimentos, metodologias de intervenção e reabilitação do Património Costeiro, por se tratar de uma atividade espontânea de transmissão oral.

Palavras-chave: Arquitetura vernacular, paleta de cor, património colombiano, rancheria Wayuu 


\section{Introducción}

En el Caribe colombiano la incidencia de la luz en la tierra y la posición geográfica juegan un papel importante en la identidad del lugar, pues intervienen y componen la estructura del espacio arquitectónico vernáculo. La arquitectura tradicional vernácula tiene una serie de características únicas y de métodos constructivos particulares que se amalgaman con su geografía inmediata y que generan un lenguaje arquitectónico, autentico y creativo que se manifiesta en la cultura de los pueblos indígenas del Caribe. Hoy en día, esta arquitectura tradicional no tiene definidos: los criterios, los procedimientos, las técnicas de intervención y rehabilitación del Patrimonio Arquitectónico Costero.

El objetivo de este trabajo es comprender cómo a través de la arquitectura en tierra y del patrón de colores, presente en la arquitectura vernácula, se obtiene una paleta cromática que es una herramienta reguladora para un uso apropiado en la arquitectura racional en los sectores de la costa colombiana; con su empleo se posibilitaría implementar una normativa en cuanto a la determinación de las paletas de color a aplicarse tanto en las nuevas construcciones residenciales como en la restauración de las antiguas en la zona costera colombiana.

Para redescubrir y promover estos diversos legados de los constructores natos de tierra se propone una metodología multidisciplinar donde en primer lugar, se reconozcan las características ambientales y cromáticas de casos similares para luego ser aplicadas a un caso en concreto. La primera parte, es el estudio del caso de la la Comunidad Indígena Jayapamana, Ranchería Wayúu en la Guajira, donde se hace un mapeo y una toma de muestras de la tierra, un estudio y un análisis, para obtener un modelo de paleta de colores. Este modelo de paleta resultante se utiliza en un caso de aplicación; concretamente en un sector del municipio de la salina: la Charca de Manaure donde se comprueba su factibilidad. Ambos lugares localizados en la región de Wopumüin, según los puntos cardinales de orientación territorial Wayúu. Esta metodología nos permite, obtener los criterios para diseñar nuevas paletas según el entorno, una previa evaluación de los impactos que podrían producir las propuestas de diseño.

La aplicación y legislación de esta paleta de colores naturales obtenida de la tierra contribuirá a la preservación del patrimonio cultural y la estética de la identidad colombiana costera. Para ello es necesario proponer normativas que regulen su aplicación sobre su patrimonio arquitectónico.

\section{Objetivo}

El objetivo de esta investigación es el reconocimiento del origen y la evolución de los valores cromáticos, generador de paletas coherentes con el entorno natural, garantizando así la conservación del patrimonio cultural de las comunidades indígenas Wayúu.

\section{Objetivos específicos:}

Identificar en la actual legislación la falta de normativas para protección de la arquitectura indígena del caribe colombiano sobre técnica o conocimiento tradicional relacionada a su restauración

Reconocer las características de la arquitectura tradicional vernácula a partir de un caso de estudio en la Comunidad Indígena Jayapamana.

Recolección de muestras en el entorno geográfico del caso de estudio de la Comunidad Indígena Jayapamana y de un caso específico en la salina las Charcas de Manaure.

Diseñar y comparar las paletas de colores de los diferentes entornos geográficos para hacer recomendaciones en la legislación para la restauración del patrimonio vernáculo en la zona costera de Colombia.

\section{Justificación}

El interés por estudiar la arquitectura vernácula Wayúu surge de la necesidad de reconocer los valores tipológicos de la arquitectura tradicional, así como sus valores compositivos, funcionales, tecnológicos y cromáticos, mediante el desarrollo de herramientas y metodologías que establezcan parámetros de intervención que contribuyan a la conservación integral y a su declaración como bien patrimonial de la nación.

Los núcleos habitacionales indígenas en Colombia han dado muestra de constantes transformaciones a raíz de las diversas dinámicas adoptadas, ya sea por la relación con la cultura mestiza o por la introducción de nuevos patrones estéticos, funcionales, compositivos, técnicos, estructurales en la arquitectura tradicional, o incluso por la implantación de nuevas formas de ordenar los elementos en la estructura habitacional colectiva, influenciadas por modelos cercanos en entornos urbanos o rurales. La suma de todos ellos distorsiona la configuración tradicional de las rancherías, de las viviendas y del núcleo construido, afectando sustancialmente la relación arquitectura tradicional - entorno natural como resultante cultural del grupo o comunidad que lo habita. [figura 1].

\section{Estado de la cuestión}

En Colombia se han centrado esfuerzos en investigaciones de arquitectura de época colonial (construcciones militares, fortificaciones, arquitectura religiosa, institucional y doméstica) de arquitectura 


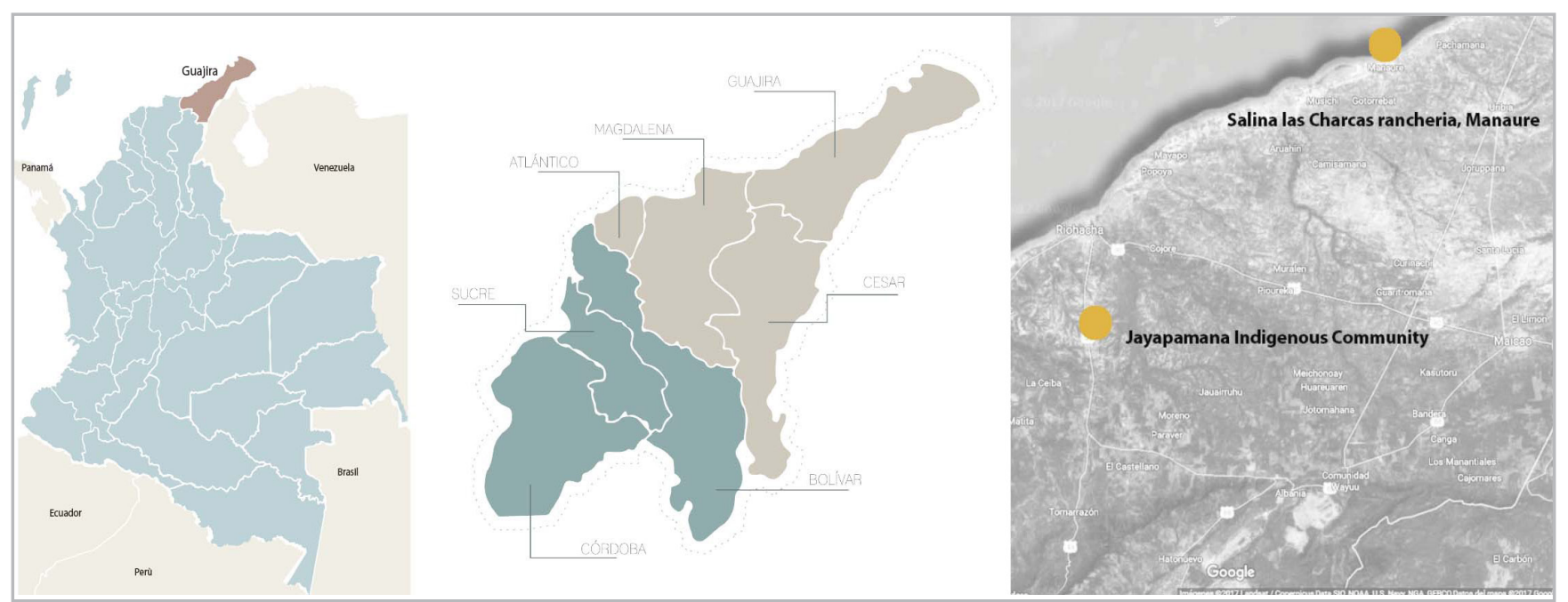

Figura 1.- Situación de las comunidades indígenas. Elaboración: propia \& Vanessa Díaz Chica..

de época republicana y movimiento moderno; sin embargo la importancia y debido reconocimiento de la arquitectura vernácula al parecer no despierta tanto interés como las tipologías más reconocidas, a pesar que se encuentran algunas investigaciones y trabajos de fin de carrera, no se registran suficientes publicaciones; la bibliografía y divulgación de la arquitectura vernácula Wayúu es escasa o insuficiente y la mayoría de casos se enfocan más a aspectos compositivos, formales y tecnológicos, dejando a un lado los valores estéticos y cromáticos; con ello determinamos la carencia de estudios técnicos del cromatismo de las tipologías arquitectónicas tradicionales específicamente en la vernácula en estudio.

\section{Legislación}

La legislación existente en Colombia es para la protección tanto de un bien cultural inmueble o mueble YA declarado patrimonio. Para que la arquitectura vernácula Colombiana sea declarada Bien de Interés Cultural, debe pasar por un proceso consignado en DECRETO NÚMERO 1080 DE 2015, donde se reglamenta el sector cultural de Colombia.

La legislación para la Gestión, Protección y Salvaguardia del Patrimonio reposa en la Ley 1185 de 2008, por la cual se modifica y adiciona la Ley 397 de 1997 (Ley General de Cultura). En su artículo $4^{\circ}$ específica: "la obligación de todo ciudadano y en especial del Gobierno Nacional la protección, la conservación, la rehabilitación y la divulgación del patrimonio cultural de la Nación que está constituido por todos los bienes materiales, las manifestaciones inmateriales, los productos y las representaciones de la cultura que son expresión de la nacionalidad colombiana, tales como la lengua castellana, las lenguas y dialectos de las comunidades indígenas, negras y creoles, la tradición, el conocimiento ancestral, el paisaje cultural, las costumbres y los hábitos, así como los bienes materiales de naturaleza mueble e inmueble a los que se les atribuye, entre otros, especial interés histórico, artístico, científico, estético o simbólico en ámbitos como el plástico, arquitectónico, urbano, arqueológico, lingüístico, sonoro, musical, audiovisual, fílmico, testimonial, documental, literario, bibliográfico, museológico o antropológico". (Garcés; López; Ariza e Isaza 2010: 9).

Dentro del Decreto 763 de 2009 por el cual se reglamentan parcialmente las Leyes 814 de 2003 y 397 de 1997, modificada por medio de la Ley 1185 de 2008, en lo correspondiente al patrimonio cultural de la Nación de naturaleza material. Dentro del Título III: Declaratoria de bienes de interés cultural (BIC) encontramos los artículos 40,41 y 44 .

En el artículo 40 en los Principios generales de Intervención encontramos tres legislaciones que se preocupan por: conservar los valores, su evolución histórica y una intervención mínima.

"1. Conservar los valores culturales del bien.

2.La mínima intervención entendida comolas acciones estrictamente necesarias para la conservación del bien, con el fin de garantizar su estabilidad y sanearlo de las fuentes de deterioro.

5. Respetar la evolución histórica del bien y abstenerse de suprimir agregados sin que medie una valoración crítica de los mismos." (Garcés; López; Ariza e Isaza 2010: 73).

En el artículo 41 de la misma sección Tipos de obras para $B I C$ inmuebles nos vuelve hablar de conservar los valores históricos:

“5. Restauración: Obras tendientes a recuperar y adaptar un inmueble o parte de éste, con el fin de conservar y revelar sus valores estéticos, históricos y simbólicos. Se fundamenta en el respeto por su integridad y autenticidad." (Garcés; López; Ariza e Isaza 2010: 74). 


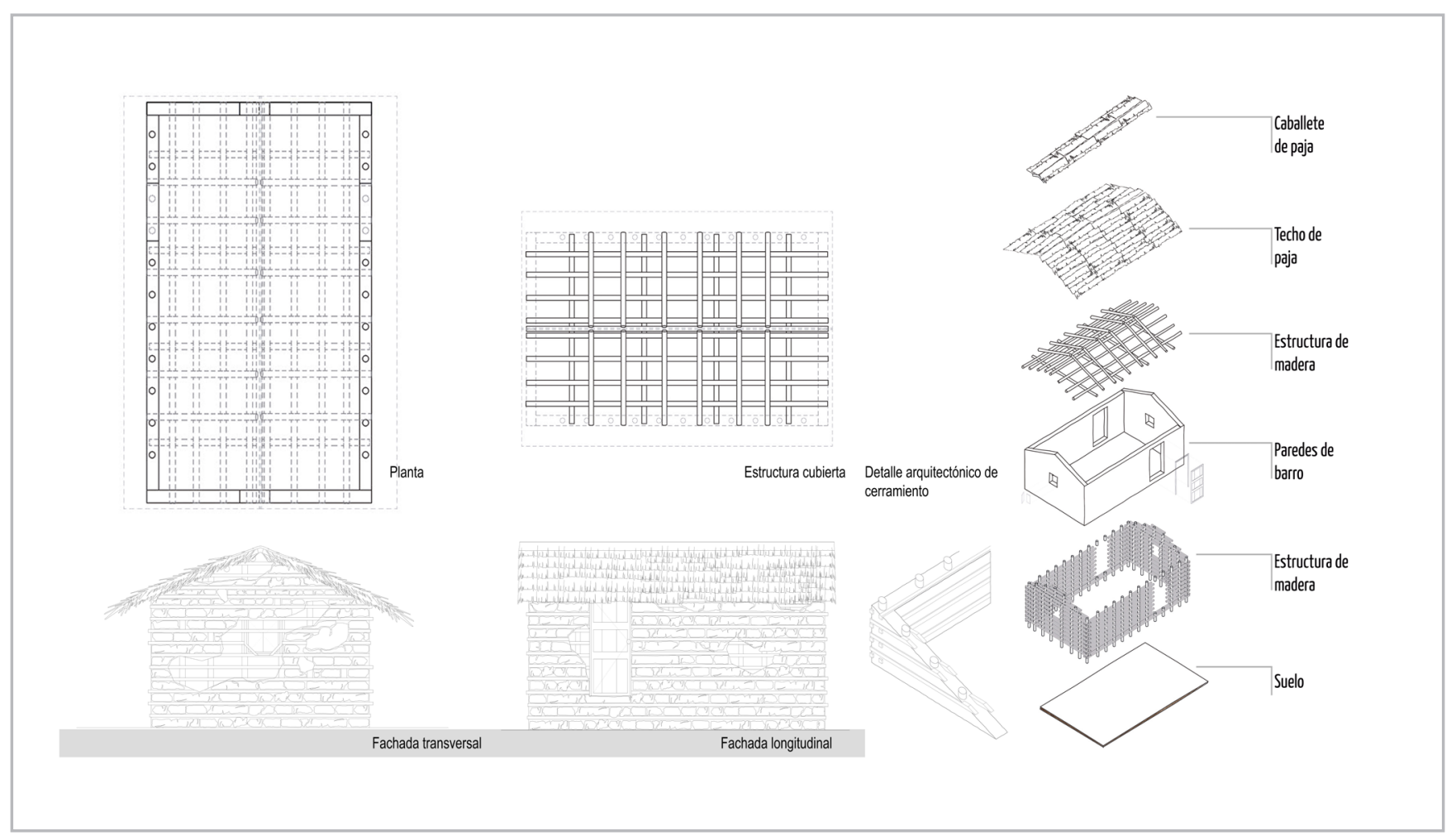

Figura 2.- Modelo de vivienda indígena. Elaboración: Propia con Omar Barboza Camargo \& Camilo Guevara Oviedo.

En el Artículo 44: Obligación de restitución de BIC por demolición no autorizada: "Si un BIC fuere demolido parcial o totalmente, o fuere intervenido sustancialmente, sin la autorización correspondiente y en contravención de las normas que obligan a su conservación, la autoridad competente procederá de manera inmediata a la paralización de dicha actividad en concurso con las autoridades de policía o locales si fuere el caso, y ordenará al propietario o poseedor la restitución de lo indebidamente demolido o intervenido según su diseño original, sin perjuicio de las sanciones previstas en la ley". (Garcés; López; Ariza e Isaza 2010: 76).

Como podemos observar los tres artículos, de La legislación para la Gestión, Protección y Salvaguardia del Patrimonio, sobre principios generales de intervención, solo mencionan aspectos y valores de tipo histórico, no hay especificaciones a nivel constructivos y mucho menos a nivel cromático para su restauración.

\section{Metodología}

Se propone una metodología interdisciplinaria que se desarrolla en dos etapas; la primera del caso de estudio la Comunidad Indígena Jayapamana, donde se hace un levantamiento para la recolección de información para la creación de sus paletas de color y su aplicación en la modelización del elemento vernáculo. La segunda etapa consiste en comparar su factibilidad y se desarrolla el caso de aplicación en la salina de las Charcas, en la que se hace un levantamiento del entorno, se crean las nuevas paletas y se completa con su nueva aplicación a través del dibujo.

\section{a) Modelo de vivienda vernácula, Piichipala}

La comunidad está organizada en clanes y a su vez en sus actividades para la subsistencia y la seguridad alimentaria. El urbanismo de una ranchería está conformado por: la vivienda, la cocina, la enramada, el corral, la escuela, las áreas de cultivo, los pozos de agua y el área de los paneles solares para la producción de la electricidad. La vivienda, la enramada, la cocina y el corral están cerca, la escuela de manera arbitraria se retira de las viviendas y por último los huertos se ubican en los límites del conjunto.

El modelo de vivienda tradicional guajira generalmente es de pequeñas dimensiones con una planta sencilla y de forma regular. Su disposición en planta parte de la división del espacio en dos habitaciones en donde cuelgan las hamacas para dormir. El pavimento es de arena descubierta. El cerramiento consiste en cuatro paredes portantes con un bajo porcentaje de vanos; solo una puerta y una ventana. Estos cerramientos poseen una estructura vinculada con la mampostería de adobe o bahareque de barro. La cubierta consta de una estructura a cuatro aguas de materiales ligeros los mismos de las paredes y está recubierta con hojas de palmas secas. Este modelo se repite en el conjunto y que tiene diferentes usos; como sitio para dormir, cocina, escuela etc. [figura 2]. Según el trabajo de grado de (Marínez; Castilla, \& Mejia: 1992) la vivienda Wayúu tiene las siguientes características [tabla 1]: 


\begin{tabular}{|c|c|c|}
\hline Nombre & Uso & Descripción \\
\hline 1. Kalpúi. & Elemento estructural de la cubierta o Sulumashi & $\begin{array}{l}\text { Puntales centrales de la estructura portante de la } \\
\text { cubierta y su entramado. Material: Madera Trupillo } \\
\text { (Prosopis juliflora) o Aipia (en lengua indígena } \\
\text { Wayuunaiki). }\end{array}$ \\
\hline 2. Súsa'a & Elemento estructural de la vivienda. & $\begin{array}{l}\text { Elementos estructurales de cerramiento del área de } \\
\text { ocupación de la vivienda llamadas patas o Súsa'a. } \\
\text { Material: Madera Trupillo (Prosopis juliflora) o Aipia } \\
\text { (en lengua indígena Wayuunaiki). }\end{array}$ \\
\hline 3. Shikipala. & Elemento estructural de la cubierta o Sulumashi. & $\begin{array}{l}\text { Caballete estructura de la cubierta. Material: Madera } \\
\text { Trupillo (Prosopis juliflora) o Aipia (en lengua } \\
\text { indígena Wayuunaiki). }\end{array}$ \\
\hline 4. Sai Wипи'u. & Elemento estructural de la cubierta o Sulumashi. & $\begin{array}{l}\text { Horquetas son los terminales de los puntales centrales } \\
\text { de la estructura de la cubierta y de las patas o Súsa'a. } \\
\text { Material: Madera Trupillo (Prosopis juliflora) o Aipia } \\
\text { (en lengua indígena Wayuunaiki) }\end{array}$ \\
\hline 5. Sipala. & Elemento Estructural de la cubierta o Sulumashi. & $\begin{array}{l}\text { Viga solera o durmiente que proporciona estabilidad } \\
\text { a las patas o Súsa'a. Material: Madera Trupillo } \\
\text { (Prosopis juliflora) o Aipia (en lengua indígena } \\
\text { Wayuunaiki). }\end{array}$ \\
\hline 6. Kulaichi. & Elemento estructural de la cubierta o Sulumashi. & $\begin{array}{l}\text { Cerchas o pares que unen el caballete con la viga } \\
\text { solera o durmiente. Material: Madera Trupillo } \\
\text { (Prosopis juliflora) o Aipia (en lengua indígena } \\
\text { Wayuunaiki) } \\
\end{array}$ \\
\hline 7. Sutujuna. & Elemento estructural de la cubierta o Sulumashi. & $\begin{array}{l}\text { Correas de amarre de la cubierta donde reposan } \\
\text { las varas de Yotojolo (Stenocereus griseus), madera } \\
\text { seca y dura que se obtiene del cactus seco. Material: } \\
\text { Tiras de cuero de chivo o lienzas de Maguey (Agave } \\
\text { Salmiana), }\end{array}$ \\
\hline 8. Nichou Supuekcna. & Carpintería. & $\begin{array}{l}\text { Puerta. Con zócalo para evitar el paso de agua y área } \\
\text { al interior de la vivienda. Material: carpintería de } \\
\text { madera. }\end{array}$ \\
\hline 9. Analaaya. & Carpintería. & Ventana. Material: carpintería de madera. \\
\hline 10. Pootshi. & Estructura de muros. & $\begin{array}{l}\text { Barro y fibras vegetales para rellenar el entramado de } \\
\text { los muros. Especie de argamasa de diversos materiales } \\
\text { de origen vegetal propios del entorno natural. }\end{array}$ \\
\hline 11. Varas de Yotojolo. & Estructura de muros y cubierta. & $\begin{array}{l}\text { Fibras de origen vegetal extraído del corazón seco de } \\
\text { la planta del Cardón. }\end{array}$ \\
\hline 12. Wunu'u Saajuna. & Elemento estructural de la cubierta. & $\begin{array}{l}\text { Vara estructural que evita que protege la cubierta de } \\
\text { las brisas. Material: Yotojolo (Stenocereus griseus), } \\
\text { madera seca y dura que se obtiene del cactus seco. }\end{array}$ \\
\hline
\end{tabular}

Tabla 1.- Modelo de vivienda indígena. Elaboración: Propia con Omar Barboza Camargo \& Camilo Guevara Oviedo.

\section{b) Paleta de color}

Con la recolección de evidencias se procedió a crear un prototipo de paleta de color. El criterio escogido para ello fue través del material, que está ligado a la función del elemento arquitectónico en la arquitectura vernácula. Así los colores se establecen en tres materiales; La hojas de palmas secas, como el elemento de la cubierta a cuatro aguas de la techumbre; la madera del Jobito o Yotojolo (Sánchez 2007), el elemento estructural del techo, de los cerramientos y de la carpintería y la tierra, para la construcción de los cerramientos de adobe y el pavimento interior.

Previo al diseño del prototipo de paleta de color, se realiza una exploración sobre los métodos de valoración 
y ordenación cromática y se decide trabajar con el sistema de ordenación cromática de Albert Munsell (1905) por ser uno de los modelos de referencias cromáticas basado en las variables de tono, valor y croma; con mayor respaldo y acreditación a nivel internacional es ampliamente implementado en diversas investigaciones de arqueología y estudios cromáticos para centros históricos patrimoniales. Además de lo anterior posee una amplia gama de tonalidades idóneas para la elaboración de cartas cromáticas históricas y para el caso de la arquitectura vernácula Wayúu ofrece una extensa gama de tonos óxidos y tierras aplicables para la ordenación cromática del entorno natural en estudio es por ello que se toma como referencia la colección: The Munsell Book of Color Nearly Neutrals Collection.

Entendiendo que la ordenación cromática de Munsell consiste en un sistema alfanumérico regido bajo sus coordenadas, identifica cada color con la referencia de letras precedidas de números, los cuales determinan el valor y la saturación (Munsell 2006). Ofrece varias colecciones donde las fichas de color están dispuestas de acuerdo al sistema de ordenación cromática. Cada ficha representa un matiz, con un total de 20 fichas en pasos de 5 matices se van mostrando de ésta manera 1100 fichas. En cada página las paletas están organizadas en función del valor y croma que Munsell establece, el valor incrementa desde la parte inferior de la página hacia arriba en incrementos de 0.5 , con un valor de 6 siendo los colores más oscuros y un valor de 9 siendo los más claros. El croma aumenta izquierda a derecha con incrementos de 0.5 con un croma de 0.5 siendo el más ligero o suave y un croma de 4 siendo el más fuerte. El matiz Munsell está impreso en la esquina superior derecha de cada página y los valores de los de las gamas están impresos en la escala vertical a la izquierda de cada página, los valores de croma están impresos en la escala horizontal en la parte inferior de la página. La forma estándar de describir la anotación del color es escribir la designación alfanumérica para el matiz de Munsell $(H)$, la designación valórica para el valor (V) y el croma (C). La escala de grises se expone en valores que van desde 6 hasta 9.5 en pasos de 0.5 y un circulo de 20 pasos de matices Ilamado Nearly Whites, en los cuales los casi blancos tienen un valor de 9.5 y cromas que van de 0.5 a 2.5 basados en el croma máximo disponible para un matiz en particular.

Siguiendo con la metodología del proyecto de investigación se definen las paletas cromáticas de los elementos analizados correspondientes al caso de estudio y a los del caso de aplicación.

\section{c) Etapa I: caso de estudio la Comunidad Indígena Jayapamana}

Las condiciones del territorio guajiro es un espacio geográfico con extensas áreas desérticas y prolongadas planicies, dada su cercanía al mar y su altitud sobre este que es casi nula.

La ranchería seleccionada para el estudio fue La Comunidad Indígena Jayapamana del clan Epieyú, ubicada a unos 20 minutos de Riohacha, en la zona media de la Guajira, colombiana. [figura 3].

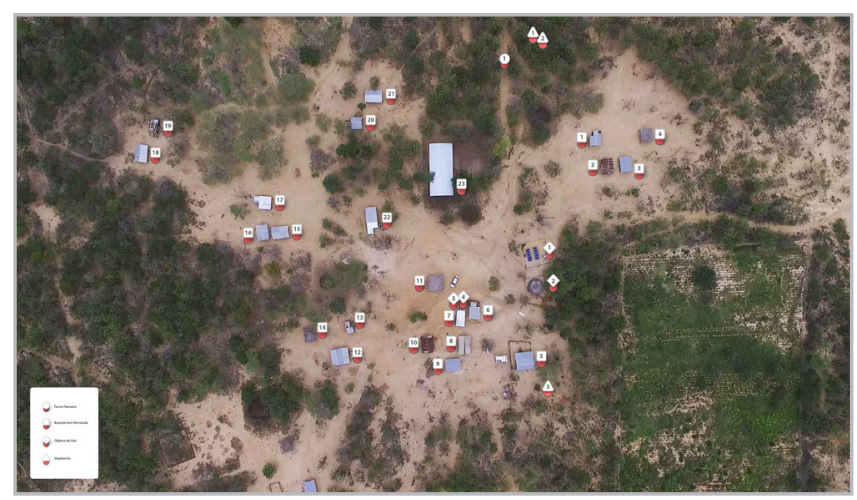

Figura 3.- Locación de la comunidad indígena Jayapamana. Elaboración: Johan Romero

\section{-1. Levantamiento del entorno}

Para la recogida de información se hizo un análisis de su entorno geográfico, proveniente de los recursos naturales, como la tierra, el paisaje, de las plantas; especialmente del cactus y del fruto del cactus; la Higoaraya (Opuntia ficus - indica). Según nos explica Arelis Pana Epieyu, indígena Wayúu, miembro del clan Epieyú', antiguamente los cultivos propios como el algodón, arroz y maíz y otras plantas como el Fique (Furcraea andina) y el Maguey (Agave Salmiana), proporcionaban color tanto en sus viviendas como en los textiles. A partir de estas apreciaciones la profesora Rossana Llanos Díaz ${ }^{2}$ recogió muestras de la vegetación, de los cactus, de la tierra y de las piedras y se hicieron registros fotográficos. Fotografías digitales para la observación del color a través de la vegetación y algunas vistas aéreas con un drone Phantom 4, que nos permitió alejarnos hasta $200 \mathrm{~ms}$ de altura, para el entendimiento del urbanismo de esta comunidad. [figura 4].

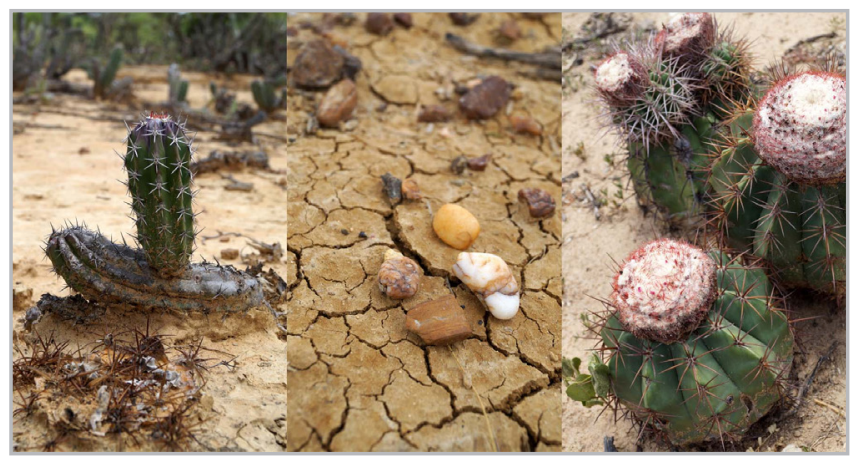

Figura 4.- Fotografías del paisaje de la comunidad Jayapamana. Elaboración: propia 


\begin{tabular}{|c|c|c|}
\hline \multicolumn{3}{|c|}{ EVIDENCIAS CASO DE ESTUDIO } \\
\hline Tipo de Evidencia & Lugar de extracción & Descripción \\
\hline 1-A Muestrario superficial de roca. & Entorno natural cauce arroyo. & Cuarzo Metafórmico I Grado. \\
\hline 1-B Muestrario superficial de roca. & Entorno natural cauce arroyo. & Chert Grado I. Sedimento Biogénico. \\
\hline 2- A Muestrario superficial de roca. & Entorno natural cauce arroyo. & Cuarzo Roca Sedimentaria. \\
\hline 2-B Muestrario superficial de roca. & Entorno natural cauce arroyo ranchería Jayapamana & Roca Volcánica Ígnea. \\
\hline 3 Muestrario a superficial de roca. & Entorno natural cauce arroyo. & Cuarzo Metafórmico II Grado Formación La Quinta. \\
\hline 4. Muestrario superficial de roca. & Entorno natural cauce arroyo. & Chert sedimentario. \\
\hline 5. Muestrario superficial de roca. & Entorno natural cauce arroyo. & Cuarzo Metafórmico. \\
\hline $\begin{array}{l}\text { 6. Muestrario de material vegetal en la } \\
\text { Vivienda vernácula. }\end{array}$ & Vivienda vernácula. & $\begin{array}{l}\text { Fibras de origen vegetal utilizada en la mezcla de barro } \\
\text { o "Pootschi“. } \\
\text { Existe diversidad de tipos de hojas de palmas secas o } \\
\text { ramas propias del entorno desértico tropical. }\end{array}$ \\
\hline $\begin{array}{l}\text { 7. Muestrario de roca en la vivienda } \\
\text { vernácula. }\end{array}$ & Vivienda vernácula. & $\begin{array}{l}\text { Mezcla de barro o "Pootschi" material de relleno del } \\
\text { entramado de los muros de la vivienda combinado con } \\
\text { hojas de palmas secas o fibras vegetales para proteger } \\
\text { la estructura de la erosión. }\end{array}$ \\
\hline $\begin{array}{l}\text { 8. Muestrario de roca en la vivienda } \\
\text { vernácula. }\end{array}$ & Vivienda vernácula. & $\begin{array}{l}\text { Mezcla de barro o "Pootschi“ material de relleno a } \\
\text { manera de argamasa de relleno del entramado de } \\
\text { muros de la vivienda. }\end{array}$ \\
\hline $\begin{array}{l}\text { 9. Muestrario de material vegetal en la } \\
\text { Vivienda vernácula. }\end{array}$ & Vivienda vernácula. & $\begin{array}{l}\text { Fibras de origen vegetal utilizada en la mezcla de barro } \\
\text { o "Pootschi“. Diversidad de tipos de hojas de palmas } \\
\text { secas o ramas propias del entorno desértico tropical. }\end{array}$ \\
\hline $\begin{array}{l}10 \text { A- Muestrario de material vegetal en la } \\
\text { Vivienda vernácula. }\end{array}$ & Vivienda vernácula. & $\begin{array}{l}\text { Vara de Yotojolo extraída del corazón seco de la planta. } \\
\text { Cardón especie de la familia de cactáceas: Pachycereus } \\
\text { pringlei. Utilizada para estructura de la cubierta. }\end{array}$ \\
\hline $\begin{array}{l}10 \text { B- Muestrario de material vegetal en la } \\
\text { Vivienda vernácula. }\end{array}$ & Vivienda vernácula. & $\begin{array}{l}\text { Fibras vegetales o especie de corteza de Trupillo } \\
\text { (Prosopis juliflora), extraída de uno de los pilares } \\
\text { estructurales de cerramiento del área de ocupación de } \\
\text { la vivienda llamadas Súsa'a o patas. }\end{array}$ \\
\hline 11. Muestrario vegetación nativa. & Vegetación entorno natural. & $\begin{array}{l}\text { Wachepa. } \\
\text { Pigmento de origen natural a base de Pali isa o Urisha } \\
\text { rojo vivo y Mashuká o Paipai polvo de tierra de tono } \\
\text { castaño. Utilizado como maquillaje y protector solar } \\
\text { para el cuerpo y rostro. }\end{array}$ \\
\hline 12. Muestrario vegetación nativa. & Vegetación entorno natural. & \\
\hline 13. Muestrario vegetación nativa. & Vegetación entorno natural. & $\begin{array}{l}\text { Vara de Yotojolo extraída del corazón seco de la planta } \\
\text { Cardón especie de la familia de cactáceas: Pachycereus } \\
\text { pringlei. Utilizada para estructura de muros y cubiertas. }\end{array}$ \\
\hline
\end{tabular}

Tabla 2.- Caso de estudio

Clasificación de las evidencias recogidas: [tabla 2]

- Muestrario superficial de roca.

- Muestrario vegetación nativa.

- Muestrario de roca en la vivienda vernácula Wayúu.

- Muestrario de material vegetal en la vivienda vernácula Wayúu

\section{Material vegetal del entorno natural}

Recolección de evidencias de manera superficial en la zona de estudio:
Las formaciones vegetales propias del entorno son especies que definen el color de la arquitectura tradicional Wayúu de la ranchería JAYAPAMANA, zona media Guajira. Naturaleza Desértica Tropical: Guamacho (Pereskia guamacho), Trupillo (Prosopis juliflora), Opuntia (Opuntia ficus-indica) y el Cardón de la familia de cactáceas el más común es el Pachycereus pringlei. Recurrentes en el uso de estructuras de muros y cubiertas.

Monte espinoso: Cactus columnares, comúnmente utilizado para cerramientos de corrales o de cocinas y arbustos espinosos para usos estructurales de muros. 
Bosque muy seco tropical: Cardón, Tuna y arbustos como el Trupillo (Prosopis juliflora), Dividivi (Caesalpinia coriaria) e Indio Desnudo (Bursera simaruba) (color rojizo). Recurrentes en el uso de estructuras de muros y cubiertas.

\section{-2. Paletas de colores}

A continuación, observamos las paletas obtenidas, básicamente en tres según el elemento arquitectónico; una para la estructura, otra para los techos y otra para los cerramientos y el suelo. [figura 5].

\section{- 3. Aplicación ranchería Jayapamana}

En el dibujo de doble proyección ortogonal e isométrico de la vivienda tradicional indígena podemos observar lo siguiente:

El pavimento y el cerramiento de adobe, habiéndose elaborado con el mismo material mantienen los mismos colores ocres de la tierra. La estructura que contienen los muros de adobe y la estructura de la cubierta elaborada con cactus, que en su mayoría es de tonos grisáceos. Y, por último; la cubierta, que está hecha de hojas de palmas secas, contiene la combinación de ambos colores. [figura 6].

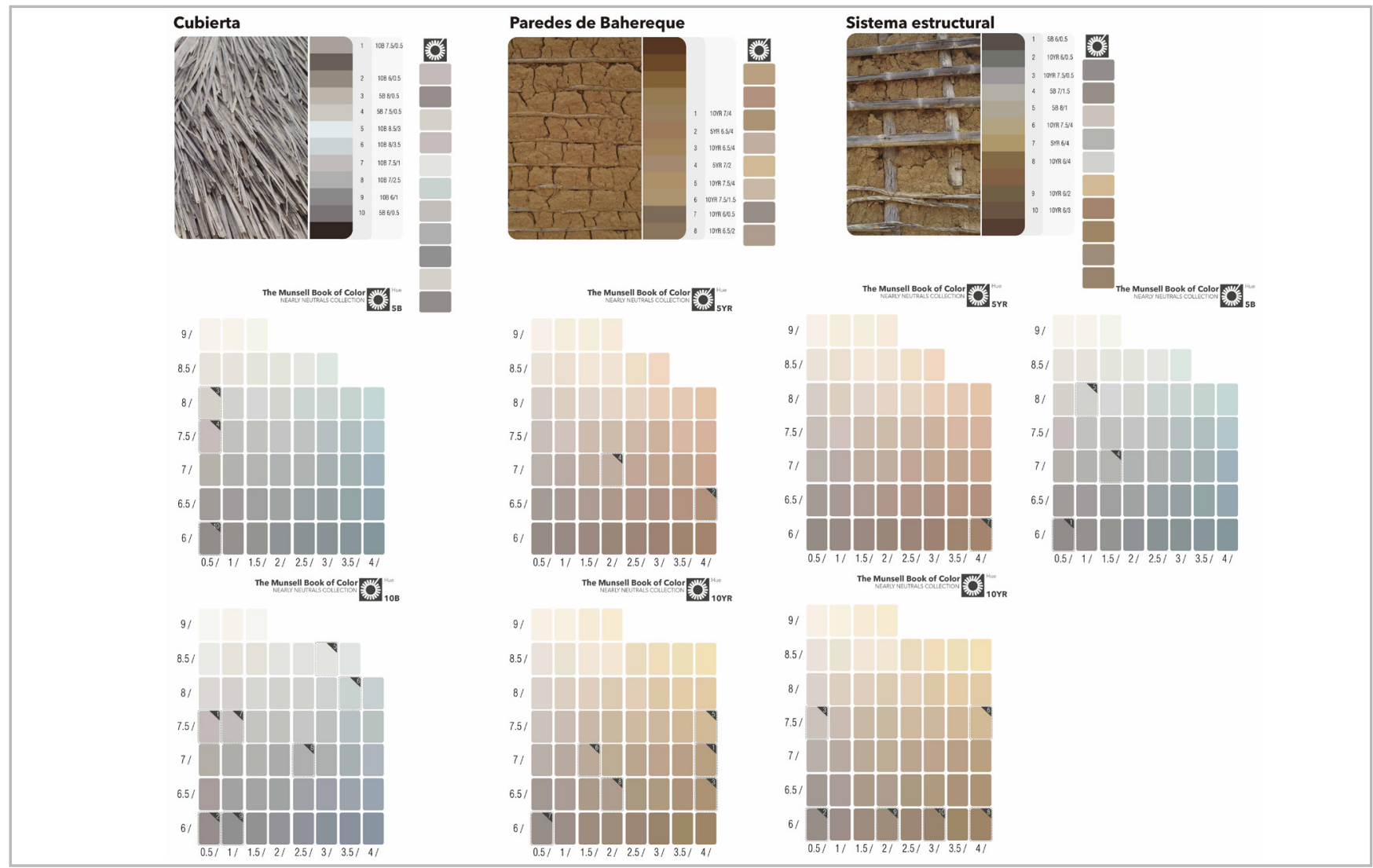

Figura 5.- Paletas de color de la Ranchería Jayapamana. Elaboración: Rossana Llanos \& Vanessa Díaz Chica

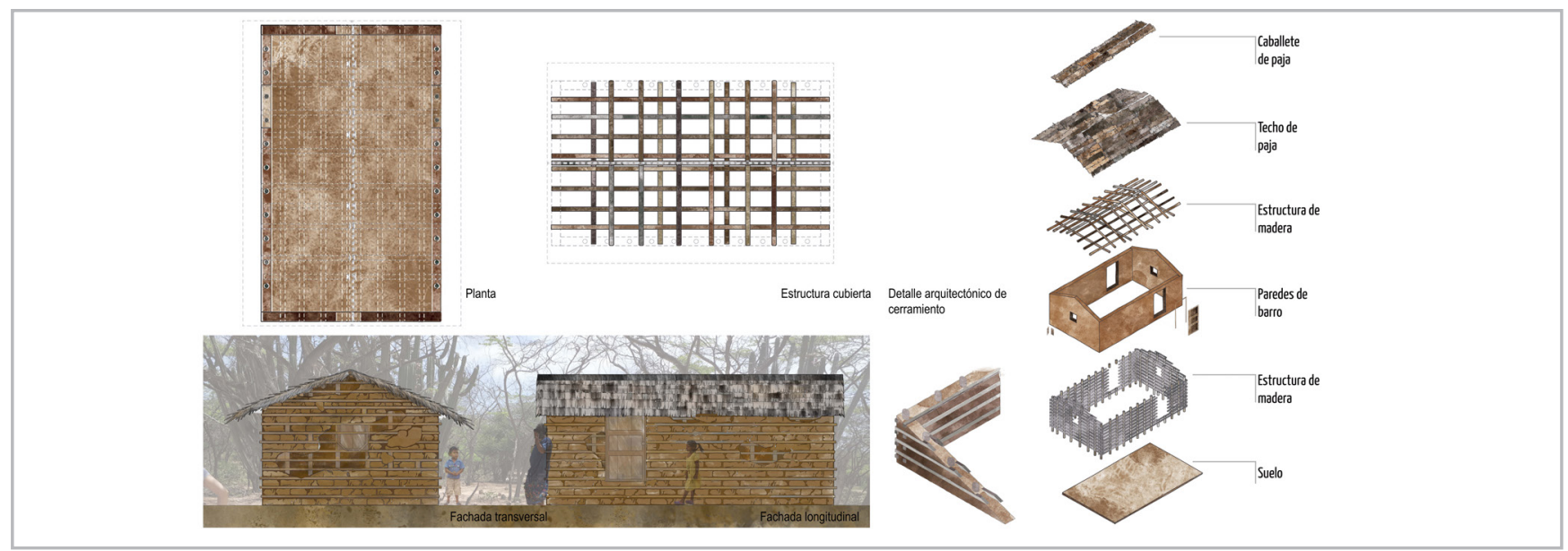

Figura 6.- Aplicación de paletas de colores en el modelo de la vivienda de la comunidad Jayapamana. Elaboración: Propia con Omar Barboza Camargo \& Camilo Guevara Oviedo. 


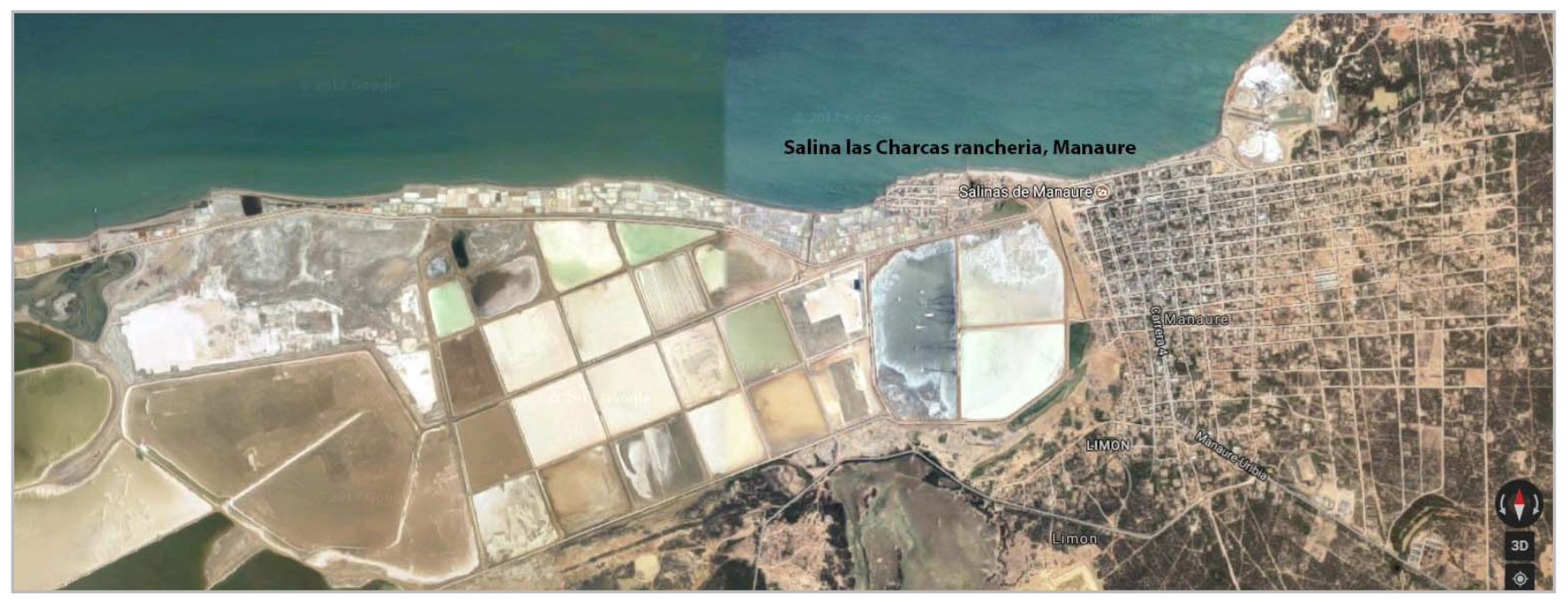

Figura 7.- Locación de la Ranchería Salina, las Charcas, Manaure. Elaboración: propia

\begin{tabular}{|l|l|l|}
\hline \multicolumn{3}{|c|}{ EVIDENCIAS CASO DE ESTUDIO } \\
\hline \multicolumn{1}{|c|}{ Tipo de Evidencia } & \multicolumn{1}{|c|}{ Lugar de extracción } & \multicolumn{1}{c|}{ Descripción } \\
\hline 14-A. Muestrario superficial & Salina las Charcas, Manaure, Guajira. & $\begin{array}{l}\text { Bivolvo. } \\
\text { Fósil de invertebrado }\end{array}$ \\
\hline 14-B. Muestrario superficial & Salina las Charcas, Manaure, Guajira & $\begin{array}{l}\text { Bivolvo. } \\
\text { Fósil de invertebrado con estructura depredación }\end{array}$ \\
\hline 15. Muestrario superficial de roca. & Salina las Charcas, Manaure, Guajira & $\begin{array}{l}\text { Halita. } \\
\text { Mineral Biogénico. } \\
\text { Sal Marina. }\end{array}$ \\
\hline
\end{tabular}

Tabla 3.- Caso de estudio

\section{d) Etapa II: Caso de aplicación ranchería Salina las Charcas, Manaure, Guajira}

Para el caso de aplicación se escogió la zona de salina las Charcas de Manaure hacia el nororiente desde Riohacha por sus características geográfica. Una zona en la media guajira colombiana que conserva sus sistemas de vida artesanal como la recolección de la sal. Esto permite que la paleta cromática conserve sus características vernáculas. [figura 7].

\section{- 1. Levantamiento del entorno: [figura 8] [tabla 3]}

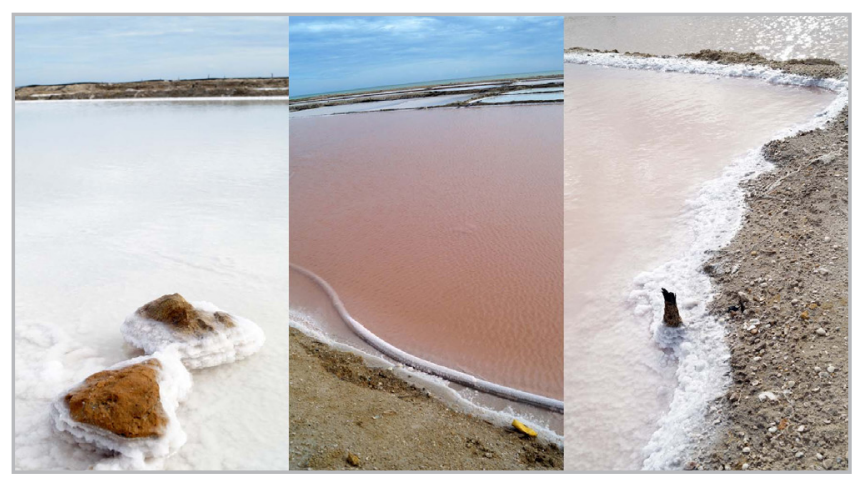

Figura 8.- Fotografías del paisaje de la Salina las Charcas. Elaboración: propia
- Muestrario superficial de roca.

-2. Paleta de color

A continuación, observamos las paletas obtenidas, básicamente en tres según el elemento arquitectónico; una para la estructura, otra para los techos y otra para los cerramientos y el suelo. El elemento estructural del techo y la carpintería,otraparaloscerramientosdeadobeyel pavimento interior y la última para la cubierta. [figura 9]. Paletas de color.

\section{-3. Aplicación en las Salinas de Manaure}

En el dibujo de doble proyección ortogonal e isométrico de la vivienda tradicional indígena se puede observar la influencia de los colores salinos.

El pavimento y el cerramiento de adobe, habiéndose elaborado con el mismo material mantienen los mismos colores pálidos y rosas de la tierra. La estructura que contienen los muros de adobe y la estructura de las cubiertas elaboradas con cactus, que en su mayoría es de tonos grisáceos claros. Y, por último; la cubierta que hecha de hojas de palmas secas contiene la combinación de ambos colores. [figura 10]. 


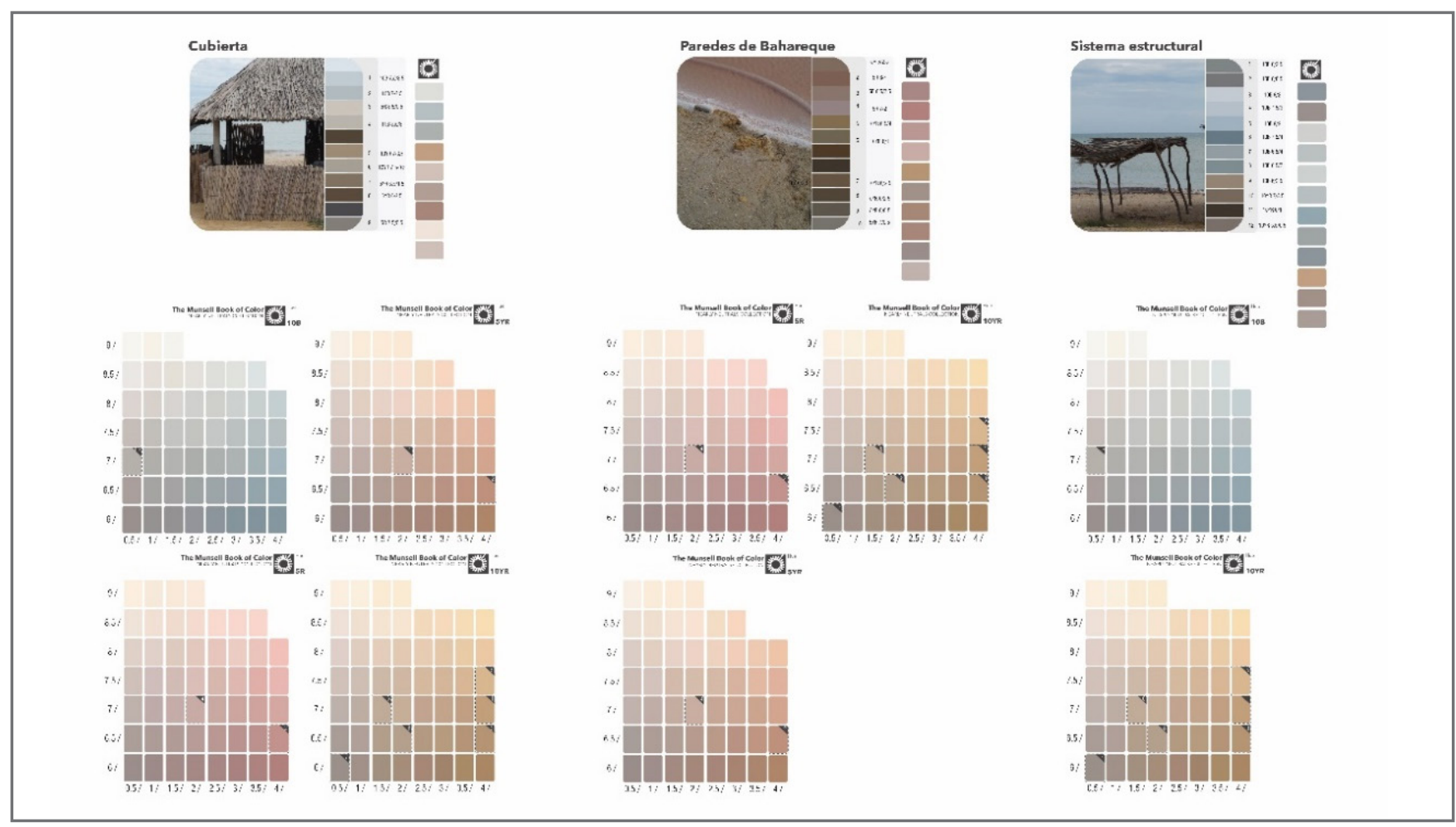

Figura 9.- Paletas de color de la Ranchería la Salina, las Charcas. Elaboración: Rossana Llanos \& Vanessa Díaz Chica.

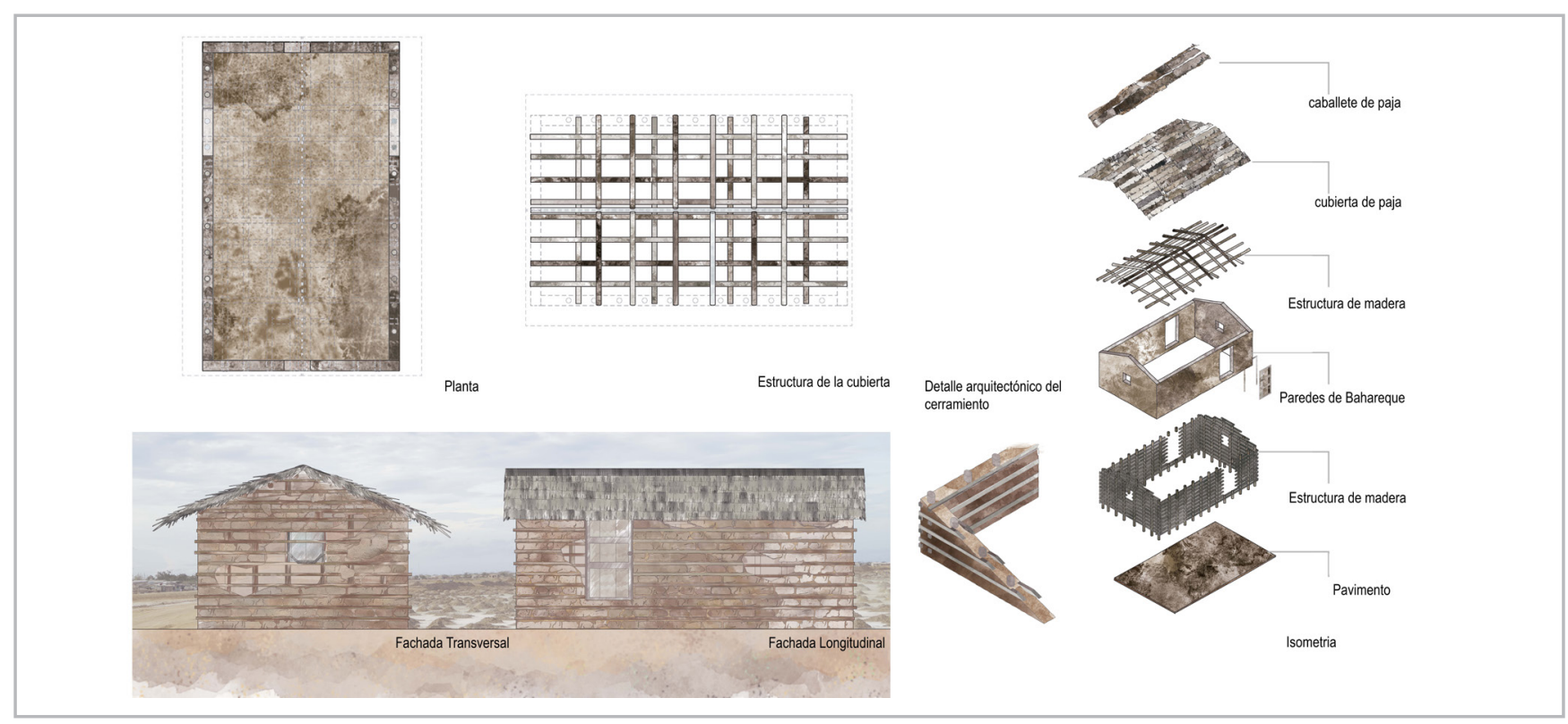

Figura 10.-Aplicación de las paletas de color en el modelo de vivienda de la Ranchería Salina las Charcas. Elaboración: Propia con Omar Barboza Camargo \& Camilo Guevara Oviedo.

\section{Propuesta de normativa para la Legislación}

Después de los trabajos realizados en el caso de estudio y el caso de aplicación consideramos que:

- Se ha de iniciar el proceso para que la arquitectura indígena guajira sea declarada Bien de Interés Cultural de la nación, así se podrán incluir normativas para su restauración en la legislación actual.

- Y en lo referente a la legislación creemos que en la Ley
1185 de 2008 Dentro del Título III: Declaratoria de bienes de interés cultural $(\mathrm{BIC})$ debe aparecer apartados donde se tome en consideración a las arquitecturas indígenas vernáculas con referencia a la técnica y al conocimiento tradicional en los dos aspectos fundamentales que la caracterizan: los valores cromáticos de la naturaleza del lugar y el sistema constructivo de las Rancherías: concretamente en la vivienda vernácula. Especificando en ella los tres materiales vegetales para los elementos constructivos que se deben respetar mínimamente a la hora de una restauración. 


\begin{tabular}{|c|c|}
\hline \multicolumn{2}{|c|}{$\begin{array}{l}\text { Valores que caracterizan a la arquitectura vernácula del Caribe } \\
\text { Colombiano: }\end{array}$} \\
\hline $\begin{array}{c}\text { Elementos Arquitectónicos } \\
\text { Constructivos }\end{array}$ & $\begin{array}{c}\text { Material Vegetal según paleta } \\
\text { cromática }\end{array}$ \\
\hline $\begin{array}{l}\text { 1. Pavimento y paredes de } \\
\text { adobe: }\end{array}$ & Tierra del lugar. \\
\hline $\begin{array}{l}\text { 2. Estructura de paredes, de } \\
\text { techos y carpintería: }\end{array}$ & $\begin{array}{l}\text { Maderas de la vegetación del } \\
\text { contexto, con vara de Yotojolo, } \\
\text { cactus. }\end{array}$ \\
\hline 3. Techo, cubiertas: & Hojas de palmas secas. \\
\hline
\end{tabular}

Tabla 4.- Valores que caracterizan a la arquitectura vernácula

\section{Conclusiones}

Las tradiciones indígenas en Colombia son manifestaciones minoritarias y se habrían de tomar medidas para su declaración y su protección. Su tradición constructiva ancestral posee valores a nivel cromático procedentes de su entorno más inmediato: la tierra y las plantas. Estos valores se han visto afectados por el impacto de compañías extractivas, colonos y misioneros, además del intercambio cultural con las Antillas (Aruba, Curazao), en donde la comercialización de los materiales ha influenciado en los tonos originales.

Por lo tanto, este trabajo presenta un acercamiento al estudio de su paleta cromática según su contexto para aportar regulaciones a la legislación vigente colombiana, para la protección y la declaración de BIC del patrimonio arquitectónico indígena guajiro.

\section{Las conclusiones son las siguientes:}

Para recoger el muestrario se definieron el contexto y la edificación vernácula. La esencia del espacio Wayúu se liga a través de la existencia y reconocimiento de su espacio territorial: su vegetación y su tierra. La vivienda Wayúu es el elemento constructivo, dentro de sus compositores de la ranchería, que alberga diferentes funciones y es el que contiene la mayor variedad de elementos arquitectónicos.

Del estudio de caso de la comunidad indígena Jayapamana y de su contexto se determinó una paleta de color basada en los elementos naturales de su emplazamiento, que a su vez establecieron los elementos arquitectónicos de la vivienda vernácula. De la vegetación se plantea la carpintería y el sistema estructural de las paredes y del techo. De la tierra: el pavimento y los muros construidos en adobe.

Teniendo como resultado el modelo de paleta del caso de estudio decidimos tomar como caso de aplicación la Ranchería Salina Las Charcas. Los resultados evidenciaron que de una manera u otra la utilización de la tierra y la vegetación siguieron definiendo el sistema constructivo. Volvimos a encontrarnos que los colores de la vegetación se concretan en la carpintería y el sistema estructural, al igual que la tierra determina su pavimento y sus paredes.
Se ratifica así el modelo de paleta cromática.

Con los modelos de paletas obtenidos del estudio se consideró que la legislación debe tener en consideración para su restauración los dos aspectos fundamentales: los valores cromáticos de la naturaleza de cada lugar y el sistema constructivo de la vivienda vernácula y que este estudio justifica acciones para iniciar los procesos para que este patrimonio sea declarado Bien de Interés Cultural.

Con ello se pretende implementar esta metodología en el estudio de otras culturas prehispánicas del Caribe colombiano que tampoco son BIC; otras comunidades indígenas como: los Tayronas, los Koguis, los Wiwas, los Kankuamos, los Ikas, etc., además de seguir con el estudio de las comunidades guajiras de Colombia y Venezuela. El estudio cromático como análisis de patrones gráficos y paletas cromáticas puede ser aplicado a otros casos de estudio, como por ejemplo los tejidos tradicionales de cada cultura: mochilas, hamacas, sombreros, calzado típico.

\section{Notas}

[1] Arelis Pana Epieyu: Directora de la Fundación Susu Wayúu con la misión y visión de visibilizar los pueblos indígenas a través del arte en Colombia. https://arelispanaepieyu.blogspot. com.es/?m=1.

[2] Rossana Llanos Díaz: Profesora del Departamento de Arquitectura, Urbanismo y Diseño de la Universidad del Norte, quien colaboró en la recolección de muestras e información para este trabajo.

\section{Bibliografía}

ALEXANDER, C. (1981). El modo intemporal de construir. Barcelona: Editorial Gustavo Gili.

ÁNGEL, C. y SÁNCHEZ C. E. (1990). El bahareque en la región del Caribe. Bogotá: Publicaciones del Servicio Nacional de Aprendizaje SENA.

CORRADINE, A. (1989). Historia de la Arquitectura colombiana Volumen -Colonia 1538-1850. Bogotá: Editorial Escala.

FONSECA M, Lorenzo. SALDARRIAGA R, Alberto. (1980). La arquitectura de la vivienda rural en Colombia Volumen 1. Bogotá: Editorial Cra. 7a. Ltda.

GARCÉS, M. LÓPEZ, C. ARIZA, E. ISAZA, J.L. (2010) “Legislación y normas generales para la Gestión, Protección y Salvaguardia del Patrimonio Cultural en Colombia. Ley 1185 y su reglamentación". Ministerio de Cultura República de Colombia. Bogotá, D. C., septiembre de 2010. http://nuevamuseologia.net/wp-content/ uploads/2014/12/Ley11854.pdf [consulta: 28/5/2018]. 
GÓMEZ, J. (2010). "Vivienda efímera urbana ¿Arquitectura Vernácula?" DEARQ: Revista de Arquitectura de la Universidad de los Andes. 7:136-143.

MARTíNEZ B, A. J., CASTILLA P, G. E., \& MEJIA S, G. (1992). Arquitectura de la comunidad Wayúu. Cartagena: Universidad Jorge Tadeo Lozano, Seccional de Caribe. Facultad de Arquitectura.

MORILLO A. (2011) Representaciones sociales de la vivienda urbana indígena: Una aproximación desde los Wayúu en Maracaibo, Venezuela.

MUNSELL, A. (2006). The Munsell Book of Color 5R-10RP. Nearly Neutrals Collection. New York: Munsell Color Services.

RAPOPORT, A. (1972). Vivienda y cultura. Barcelona: Gustavo Gili.

SÁNCHEZ GAMA, C. (2007) "La arquitectura de tierra en Colombia, procesos y culturas constructivas" 20, 2: 242-255.

\section{Autor/es}

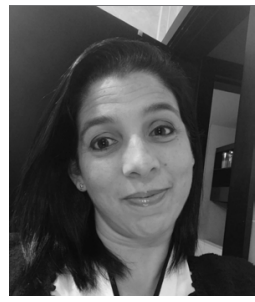

\section{Mariolly Dávila Cordido}

madacor@alumni.uv.es

Escuela de Arquitectura. Universitat de València.

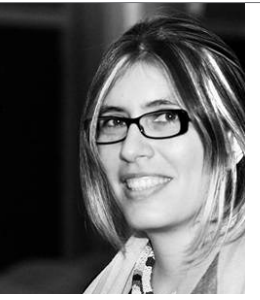

\section{Caterina Macchi Jánica}

caterinamacchi@gmail.com

Escuela de Arquitectura, Urbanismo y Diseño Universidad Autónoma del Caribe (Colombia)

Diseñadora Gráfica en 2001 Universidad Jorge Tadeo Lozano Seccional Caribe (Cartagena, Colombia). Estudió en Florencia (Italia) diseño de joyas en el Studio Arrais en el año 2003, y joyería básica y talla de piedras en la escuela Macorlyn Joyeros en la ciudad de Bogotá. Se ha dedicado a labores académicas e investigativas desde los talleres de diseño en diferentes instituciones educativas de la región Caribe, desde el año 2002 en la Universidad Jorge Tadeo Lozano y en Barranquilla (Cartagena), en LaSalle College y también como directora del Programa de Diseño Gráfico de la Facultad de Arquitectura, Arte y Diseño de la Universidad Autónoma del Caribe y en la Universidad del Norte desde el 2007, vinculación que continúa desde sus labores pedagógicas al servicio de la Escuela de Arquitectura, Urbanismo y Diseño como Docente de Tiempo Completo. Ha realizado en Italia algunos Workshops: TM Italia, Ascoli "La cocina Eco-Emocional". Industria IFI, "Coffee Experience Design". Indesit Company "Electrodomésticos Eco-Sensibles. FAAM Spa "Eco-Design y nuevos escenarios aplicados a baterías a litio". Eusebi Arredamenti Eco-Sistemas Domésticos. Revolution S.p.A. "Living Design".
Arquitecto y Doctorado en Comunicación Visual en Arquitectura y Diseño por la Escuela Técnica Superior de Arquitectura de Barcelona de la UPC de Barcelona, España. Postgrados en Nivelación en Acondicionamientos y Servicios y Nivelación en Diseño y, Cálculo de Estructuras Arquitectónicas, ambos de la Fundación UPC. Barcelona, España, y Arquitecto por la Facultad de Arquitectura y Urbanismo (UCV) de Caracas, Venezuela. Ha participado en diferentes concursos de pintura. En 1986 gana el premio nacional de pintura en el Concurso: "Pequeños Pinceles" de la Radio Nacional de Venezuela. En 1999 obtiene del programa de becas de la Fundación Gran Mariscal de Ayacucho en Venezuela el crédito educativo para la realización del doctorado en Comunicación Visual en Arquitectura y Diseño, Representación Gráfica Arquitectónica. Junto a los estudios de arquitectura, desde 1996 hasta 2012, ha desarrollado su carrera profesional colaborando en numerosas oficinas de arquitectura como Project manager, entre Caracas y Barcelona. La línea de investigación; entre proyectos de investigación, artículos y participación en congresos está relacionada con la proyección del espacio arquitectónico y su visualización tridimensional través de diferentes metodologías y herramientas que incluyen la geometría y realidades virtuales durante el proceso del diseño o en la recuperación de las edificaciones desaparecidas de valor patrimonial.
Artículo enviado el 14/11/2017 Artículo aceptado el 28/09/2018 